Uffink, J. 2001. Bluff your way in the second law of thermodynamics. Studies in History and Philosophy of Science Part B: Studies in History and Philosophy of Modern Physics 32: 305-94.

Uffink, J. 2007. Compendium of the foundations of classical statistical physics. In Philosophy of Physics, eds. J. Butterfield and J. Earman. Amsterdam: North Holland.

Uhlenbeck, G.E. and G.W. Ford. 1963. Lectures in Statistical Mechanics. Providence, RI: American Mathematical Society.

van Lith, J. 1999. Reconsidering the concept of equilibrium in classical statistical mechanics. Philosophy of Science 66: 107-18.

\title{
Blur and perceptual content
}

\section{Bence Nanay}

\section{Introduction}

Intentionalism about visual experiences is the view according to which the phenomenal character of a visual experience supervenes on the content of this experience. ${ }^{1}$ One of the most important counterexamples to intentionalism is the following. Seeing a fuzzy contour clearly and seeing a sharp contour blurrily have different phenomenal character but the same content (the objection was first raised by Boghossian and Velleman (1989)). Hence, the phenomenal character of perceptual experience cannot supervene on the content of this experience as in this example two perceptual experiences have the same content and yet they have different phenomenal character.

I argue that if we accept a simple and not particularly controversial way of thinking about perceptual content, then we have no reason to suppose that these two experiences have the same content. I then compare my proposal about blur with Michael Tye's seemingly similar account and point out that they are in fact very different, so much so that my proposal can give adequate answers to the two most important objections Tye's account faces.

\section{Blur and determinacy}

Compare the following two experiences. First, I am looking at a fuzzy contour, say, a blurry photograph and I see the photograph clearly. Second, I am looking at a clear contour, but I see this contour blurrily (because I don't have my glasses on). The original objection to intentionalism was that these two experiences have the same content but different phenomenal character.

1 I will assume throughout the paper that perceptual experiences have content. This is not an uncontroversial assumption these days. See a series of papers by Craig French on how one can talk about blurry vision in a naïve realist framework (French 2014, 2015). 
Hence, the phenomenal character of perceptual experiences cannot supervene on their content.

There are two ways of attacking this objection to intentionalism. One would be to deny that the two experiences have different phenomenal character (see, e.g., Schroer 2002). I will not do this: disagreements about phenomenology are notoriously difficult to settle, so those who want to defend intentionalism are better off accepting for the sake of the argument that the two experiences differ in their phenomenal character.

The other strategy is to deny that they have the same content. There are many different ways of doing this (just a few: Allen 2013; Crane 2006; Dretske 2003; Kelly 2010; Pautz 2010; Tye 2002, 2003), but regardless of which of these versions we consider, the success of this general strategy clearly depends on how we think about perceptual content.

In the next section, I outline a straightforward account of perceptual content. Then in Section 4, I argue that if we accept way of thinking about perceptual content, blur poses no problem for intentionalism. In Section 5, I contrast my account with Michael Tye's.

\section{Perceptual content}

Consider the following, very simple, and not particularly controversial, way of thinking about perceptual content (see, e.g., Kulvicki 2007; Nanay 2010, 2015a, 2015b). Our perceptual apparatus attributes various properties to various parts of the perceived scene. Perceptual content is partly constituted by the sum total of the properties attributed to the perceived scene. ${ }^{2}$ The question is what kind of properties are being attributed to the perceived scene.

One way of characterizing the relation between properties is the determinable-determinate relation (Funkhouser 2006; Johnston 1921). To use a classic example, being red is determinate of being coloured, but determinable of being scarlet. There are many ways of being red and being scarlet is one of these: for something to be scarlet is for it to be red, in a specific way. If something is red, it also has to be of a certain specific shade of red: there is no such thing as being red simpliciter.

The determinable-determinate relation is a relative one: the same property, for example, of being red, can be the determinate of the determinable being coloured, but the determinable of the determinate being scarlet. Thus, the determinable-determinate relation gives us hierarchical ordering of properties

2 I say 'partly constituted' in order to deliver on my promise that this account of perceptual content is not particularly controversial as it would allow for the potential role of the particular these properties are attributed to as well as for the 'mode of presentation' of these properties (if these are to be distinguished from the attributed properties). 
in a given property-space. Properties with no further determinates, if there are any, are known as super-determinates.

Some of the properties we perceptually attribute to the perceived scene are determinates or even super-determinates. Some others, on the other hand, are determinable properties. We know that our peripheral vision is only capable of attributing extremely determinable properties. But even some of the properties we perceptually attribute to the objects that are in our fovea can be determinable.

It has been argued that this way of thinking about content (with the additional assumption that perceptual attention makes the attended property more determinate) helps us to explain away some other influential counterexamples to intentionalism: ones that have to do, one way or another, with attention (Nanay 2010, 2011; Stazicker 2011). My aim here is to show that if we accept this, again, not particularly controversial, account of perceptual content, then we have a simple way of handling the alleged counterexample to intentionalism that blurry vision poses.

\section{Blur and determinacy}

Remember the two experiences that pose a problem for intentionalism: looking at a blurry photograph clearly and looking at a clear contour blurrily. If we accept the account of perceptual content I outlined in the last section, then these two experiences do not have the same content. When I see a fuzzy contour clearly, I attribute determinate (maybe superdeterminate) properties to the fuzzy contour. When I see the sharp contour blurrily, in contrast, I attribute determinable properties to the sharp contour. The content of these two perceptual episodes are very different.

Let us proceed more slowly and consider the ways in which perceptual states can represent the property of having a certain spatial location. A perceptual state can attribute determinable or determinate property-instances of having a spatial location. When I am looking at (and attending to) the spacebar of my laptop right in front of me, my perceptual state attributes a highly determinate (maybe even super-determinate) spatial location property to this object. But on many other occasions (say, when I am too far away from it or when I only catch a glimpse of it in the periphery of my visual field), my perceptual state will attribute a determinable spatial location property to this object: the property of approximately what spatial region it is located in.

My claim is that the difference between seeing a fuzzy contour clearly and seeing a sharp contour blurrily is a difference of this kind. When I see a fuzzy contour clearly, my perceptual state attributes a specific, highly determinate property to this contour. It may be blurry but I see it as being at a specific, highly determinate spatial location. But when I see the sharp contour blurrily, 
my perceptual state attributes a determinable spatial location property to this contour. I do not see this contour as being at a specific, highly determinate spatial location. I see it as being at an approximate, determinable spatial location: as being somewhere in the blurry-looking spatial region.

In short, the difference between the two experiences is a difference of the determinacy of the spatial location properties perceptually attributed to the scene. When I see the fuzzy contour clearly, these properties are determinates. When I see the sharp contour blurrily, they are determinables. As perceptual content is partly constituted by the sum total of the properties perceptually attributed to the perceived scene, the perceptual content in the two scenarios are clearly different. Hence, the fact that the two experiences have different phenomenal character (if they do) does not constitute a counterexample to intentionalism.

\section{Determinacy versus 'failure to comment'}

Michael Tye proposed a seemingly similar explanation of how the phenomenon of blurry vision is consistent with intentionalism. I aim to point out that my account is importantly different from Tye's, so much so that it can give adequate answers to the two most important objections to Tye's explanatory scheme.

According to Tye, 'in seeing blurrily, one undergoes sensory representations that fail to specify just where the boundaries and contours lie. Some information that was present with eyes focused is now missing' (Tye 2002: 147-148). In short, 'one's visual experience ... makes no comment on where exactly the boundaries lie' (Tye 2003: 18).

There are some similarities between my account and Tye's inasmuch as both deny that blurry vision attributes determinate properties. But there is a crucial ambiguity in Tye's account. Sometimes he seems to deny that any properties are represented (if we interpret failure to comment to mean failure to represent). But some other times, he seems to allow for some represented properties, for example when he talks about 'less definite information about surface depth, orientation, contours, and so forth' (Tye 2002: 80). Sometimes, for example, when he says that 'my visual experience is silent on the precise locus (if any) of the edges' (Tye 2003: 19), he seems to endorse the first, no representation at all interpretation. The second quote in the previous paragraph (from Tye 2003), in contrast, talks about failure to comment on 'where exactly the boundaries lie' (my emphasis). This sounds more similar to the view I defend.

My aim here is not a close analysis of Tye's text. If his official view is that blurry vision fails to comment on the exact boundaries, but does comment on the approximate (that is, determinable) boundaries, then his view is compatible with mine and my view could be thought of as an elaboration of his. 
If his official view is that blurry vision fails to comment on the boundaries at all, exact or approximate (which seems to be the standard interpretation of Tye's view, see French 2014, 2015; Pace 2007; Smith 2008), then, I will argue, my view is preferable to Tye's. In fact, my account can easily answer the two most important objections to Tye's proposal (interpreted as a proposal about failure to comment tout court).

The first objection to Tye's proposal (again, interpreted as a proposal about failure to comment tout court) is that according to Tye, the difference between seeing a fuzzy contour clearly and seeing a sharp contour blurrily is not a difference of what is represented but of what is not represented (Smith 2008: 206-207). Thus, strictly speaking, it is not a representational difference that the intentionalist would need in order to argue that phenomenal character supervenes on perceptual content (see Pautz 2010 for a different assessment of whether this explanation of blur is consistent with bona fide intentionalism).

If we accept my proposal, then the difference between seeing a fuzzy contour clearly and seeing a sharp contour blurrily is in fact a representational difference: a difference of what properties are being attributed to the perceived scene. In the former case, these perceptually attributed properties are determinate, whereas in the latter case, they are determinable.

The second objection is that if we accept Tye's analysis, seeing something blurrily and seeing something in the periphery of one's visual field would have the same content: it is true of both experiences that they 'fail to comment on where exactly the boundaries lie'. But these two experiences with the same content have different phenomenology. Hence, the original objection can be now raised in the case of these two experiences: seeing a sharp contour blurrily and seeing it in the periphery of one's visual field. They have the same content, but clearly different phenomenology, which means that phenomenology does not supervene on perceptual content: intentionalism is false (a version of this argument is given in Pace 2007: 334-335).

According to my proposal, there is a clear difference between the content of these two experiences. When I see a sharp contour blurrily, the spatial location property that is perceptually attributed to this contour is determinable. But, as long as I am attending to this contour, a number of other properties that are also perceptually attributed to this contour, for example, its colour property, will be determinate (or even super-determinate). In the case of peripheral vision, in contrast, our perceptual system can only attribute determinable properties to the objects seen in the periphery of one's visual field - not just determinable spatial location properties, but determinable colour properties, shape properties, etc. Hence, we have a clear representational difference between blurry vision and peripheral vision: the former attributes determinable spatial location properties, but determinate colour-, shape-, etc. properties, the latter attributes only determinable 
properties. The perceptual contents of these two perceptual episodes are very different.

\title{
Funding
}

This work was supported by the ERC Consolidator grant [726251], the FWO Odysseus grant [G.0020.12N] and the FWO research grant [G0C7416N].

Centre for Philosophical Psychology University of Antwerp D 413, Grote Kauwenberg 18 Antwerp 2000, Belgium

\author{
Peterhouse College \\ University of Cambridge \\ Cambridge CB2 1RD, UK \\ bn206@cam.ac.uk \\ bence.nanay@uantwerpen.be
}

\section{References}

Allen, K. 2013. Blur. Philosophical Studies 162: 257-73.

Boghossian, P.A. and J.D. Velleman. 1989. Colour as a secondary quality. Mind 98: 81-103.

Crane, T. 2006. Is there a perceptual relation? In Perceptual Experience, eds. T. Gendler and J. Hawthorne, 126-46. Oxford: Oxford University Press.

Dretske, F. 2003. Experience as representation. Philosophical Issues 13: 67-82.

French, C. 2014. Naïve realist perspectives on seeing blurrily. Ratio 27: 393-413.

French, C. 2015. Idiosyncratic perception. Philosophical Quarterly 66: 391-9.

Funkhouser, E. 2006. The determinable-determinate relation. Nous 40: 548-69.

Johnston, W.E. 1921. Logic. Part I. Cambridge: Cambridge University Press.

Kulvicki, J. 2007. Perceptual content is vertically articulate. American Philosophical Quarterly 44: 357-69.

Nanay, B. 2010. Attention and perceptual content. Analysis 70: 263-70.

Nanay, B. 2011. Ambiguous pictures, attention and perceptual content. Phenomenology and the Cognitive Sciences 10: 557-61.

Nanay, B. 2015a. Perceptual content and the content of mental imagery. Philosophical Studies 172: 1723-36.

Nanay, B. 2015b. Perceptual content. In Oxford Handbook of the Philosophy of Perception, ed. M. Matthen, 153-67. Oxford: Oxford University Press.

Pace, M. 2007. Blurred vision and the transparency of experience. Pacific Philosophical Quarterly 88: 328-54.

Pautz, A. 2010. Why explain visual experience in terms of content? In Perceiving the World: New Essays on Perception, ed. B. Nanay, 254-310. New York: Oxford University Press. 
Schroer, R. 2002. Seeing in all clearly: the real story on blurry vision. American Philosophical Quarterly 39: 297-301.

Smith, A.D. 2008. Translucent experiences. Philosophical Studies 140: 197-212.

Stazicker, J. 2011. Attention, visual consciousness and indeterminacy. Mind \& Language 26: $156-84$.

Tye, M. 2002. Representationalism and the transparency of experience. Nous 36:13751.

Tye, M. 2003. Blurry images, double vision and other oddities: new problems for representationalism? In Consciousness: New Philosophical Perspectives, eds. Q. Smith and A. Jokic, 7-32. Oxford: Clarendon Press.

\section{If time travel to our location is possible, we do not live in a branching universe}

JAMES NorTON

This article argues for the following disjunction: either we do not live in a world with a branching temporal structure, or backwards time travel ${ }^{1}$ is nomologically impossible, given the initial state of the universe, or backwards time travel to our space-time location is impossible, given large-scale facts about space and time. A fortiori, if backwards time travel to our location is possible, we do not live in a branching universe.

Horwich (1987: 1) gestures towards an interesting argument for the conclusion that there is no backwards time travel in the actual world. For, if there is some future time, $t$, at which the requisite technology is developed and subsequently used, we would expect to see, around here, time travellers originating from $t$. We see no such time travellers, and thus there is no actual time travel. ${ }^{2}$ Call this (following Smith 2016) the 'where are the time travellers?' argument. Fulmer (1980) articulates, but declines to endorse, the same style of argument. Reinganum (1986) argues in a similar vein that if there is

1 When I talk of backwards time travel, I have in mind the interesting kind, where future individuals can travel back and interact with past individuals.

2 'If the necessary technology will ever become available, why haven't we encountered visitors from the future?' (1987: 1). In chapter 7 of the same volume, Horwich presents his better-known argument against time travel: namely, that backwards time travel would result in 'an endless string of improbable coincidences' (1987: 123). This latter argument is given a thorough treatment in Smith 1997 and is not my focus here. Likewise, while Hawking (1992: 610) notes that ' $[\mathrm{t}]$ here is also strong experimental evidence in favor of the conjecture [that there is no actual time travel] from the fact that we have not been invaded by hordes of tourists from the future', his primary argument - that there is no actual time travel due to the nomological impossibility of closed time-like curves - is not my focus. 